Introduction Female sex workers (FSW) may experience barriers to accessing sexual health services. Appropriate treatment for STIs is paramount to prevent the spread of antibiotic resistant infections. We aimed to understand how healthcare access might impact self-treatment and antibiotic use for genitourinary symptoms among FSWs in Tijuana, Mexico.

Methods 282 FSW participants were tested for STIs and underwent an interview. APTIMA COMBO 2 (Hologic, Inc.) was used to diagnose cervicovaginal Neisseria gonorrhoeae (NG) infection. We summarised data and used logistic regression to assess associations between self-medication (use of pills and/or injections that were not prescribed by a doctor or nurse) for genitourinary symptoms and prior HIV test (a proxy for accessing sexual health services), income and STI history. Genitourinary symptoms included dysuria, vaginal discharge, vaginal bleeding between periods, pelvic pain, or dyspareunia.

Results 282 FSW participants were recruited in Tijuana. Of the 263 with test results, $41(15.6 \%)$ tested positive for NG, of which $23(56.1 \%)$ endorsed current genitourinary symptoms. Of the 282 total participants, $60(21.3 \%)$ reported that they had ever self-medicated to treat genitourinary symptoms. Never having an HIV test $(\mathrm{OR}=2.3$ (95\% CI: 1.3, 4.3)) and reporting lower income $(<\mathrm{MX} \$ 3500$ vs. $\geq \mathrm{MX} \$ 3500)$ $(\mathrm{OR}=2.2 \quad(95 \% \mathrm{CI}: 1.1,3.6)$ were associated with reporting ever self-medicating for treatment of genitourinary symptoms. In addition, those that reported they had ever had an STI $(\mathrm{n}=83 \quad(29.4 \%), \quad \mathrm{OR}=2.7 \quad(95 \% \mathrm{CI}: 1.5,4.8))$ were more likely to report self-medication for treatment of genitourinary symptoms.

Conclusion We found a high prevalence of NG infection among a cohort of FSWs in Tijuana, Mexico. The high prevalence and past medication use without a prescription point to a potential for emerging NG antibiotic resistance in this setting. Barriers to access for sexual healthcare may drive the use of medications without a prescription. Additionally, those with a history of STIs may be self-treating for familiar symptoms.

\section{P4.28 STIGMA AND DISCRIMINATION EXPERIENCES IN HEALTH CARE SETTINGS MORE EVIDENT AMONG TRANSGENDER PEOPLE THAN MALES HAVING SEX WITH MALES (MSM) IN INDONESIA, MALAYSIA, THE PHILIPPINES AND TIMOR LESTE: KEY RESULTS}

RN Cortes. The Philippine Ngo Council on Population, Health and Welfare, INC., Pasay City, Philippines

\subsection{6/sextrans-2017-053264.525}

Introduction A four-country study was conducted by the ISEAN-Hivos Program (Global Fund regional AIDS grant) which aims to provide information on the status of stigma and discrimination (SAD) among males having sex with males (MSM) and transgender people in health care settings (including HIV/AIDS services) using a questionnaire based on the forms of SAD described in the Stigma and Discrimination Index Questionnaire.

Methods The study's questionnaire described SAD in terms of the respondents' self-reported perception of: 1 . Refusal of health care services, 2. Physical maltreatment, 3. Verbal maltreatment, and 4. Provision of health care service below standards. A total of 2409 respondents, 30\% $(n=719)$ of whom are self-identifying male-to-female trans persons, participated in this study. There were 264 trans respondents from Indonesia, 204 in the Philippines, 174 in Malaysia, and 77 in Timor Leste.

Results Overall, the results indicate that significantly more trans people experienced SAD in health care settings compared to MSM. Verbal maltreatment was the most commonly experienced $(24.26 \%)$, followed by receiving a perceived low quality of health service (22.57\%), being refused access to health care services $(18.23 \%)$ and lastly, physical maltreatment (18.21\%). There were proportionately more SAD experiences reported by trans people in Timor Leste (41.06\%), followed by Malaysia (32.67\%), Philippines (7.47\%) and Indonesia $(2.0 \%)$.

Conclusion The result of the SADS suggests that there is a wide variation across the four countries in terms of trans people experiencing stigma and discrimination. Verbal maltreatment is the topmost common form of SAD among trans people. The study indicates that trans' personal experiences of SAD are more frequent than MSM. Also, about one third of the trans people continue to experience SAD. Almost $60 \%$ of the trans people, however, did nothing to address SAD. SAD still exists in health care settings, which needs support for more interventions to significantly decrease, if not totally eradicate SAD in its many forms.

\section{P4.29 THE VALUE OF BUILDING SOCIAL NETWORKS AMONG NIGERIAN MEN WHO HAVE SEX WITH MEN AFFECTED BY SEXUAL STIGMA: A QUALITATIVE STUDY OF BARRIERS AND BENEFITS}

${ }^{1}$ Cristina Rodriguez-Hart, ${ }^{2}$ Cory Bradley, ${ }^{2}$ Danielle German, ${ }^{3}$ Ifeanyi Orazulike, ${ }^{4}$ Blessing Kayode, ${ }^{5}$ Hongjie Liu, ${ }^{6}$ Trevor A Crowell, ${ }^{2}$ Stefan Baral, ${ }^{1}$ Man Charurat, ${ }^{1}$ Rebecca $G$ Nowak. 'Institute of Human Virology University of Maryland School of Medicine, Baltimore, USA; ${ }^{2}$ Johns Hopkins Bloomberg School of Public Health, Baltimore, USA ${ }^{3}$ International Centre on Advocacy and Rights to Health, Abuja, Nigeria; Institute of Human Virology Nigeria, Abuja, Nigeria; ${ }^{5}$ University of Maryland School of Public Health, College Park, USA; ${ }^{6}$ U.S. Military Hiv Research Program, Walter Reed Army Institute of Research, Silver Spring; Henry, Bethesda, USA

\subsection{6/sextrans-2017-053264.526}

Introduction Sexual stigma affecting men who have sex with men (MSM) in Nigeria may contribute to acquisition and onward transmission of HIV and other sexually transmitted infections (STIs). Prior analysis found stigma to be associated with increased HIV and STI prevalence and larger MSM social network size. The aim of this study was to explore the association between stigma and MSM social networks.

Methods From March 2013 to February 2016, the TRUST/ RV368 study recruited 1,480 MSM in Abuja and Lagos, Nigeria into a prospective cohort that provides HIV and STI diagnosis and treatment. From this parent study, 15 semistructured in-depth interviews were conducted with participants who experienced elevated stigma. Interviews were transcribed and coded using thematic analysis.

Results The key themes for how sexual stigma may have led to greater vulnerability for HIV and STIs were: financial vulnerability and emotional trauma, constrained opportunities to share HIV and STI resources, and internet use. Participants described financial exploitation and emotional trauma as common consequences of stigma that at times was associated with condomless sex and transactional sex. Fear of stigma constrained MSM's willingness to openly socialise, which may have limited exposure to sexual health services, as MSM social 